\title{
On the nature of dust clouds in the region towards M 81 and NGC 3077
}

\begin{abstract}
A. Heithausen ${ }^{\star}$
I. Physikalisches Institut, Universität Köln, Zülpicher Str. 77, 50937 Köln, Germany

e-mail: aheithau@uni-koeln.de

Received 9 August 2011 / Accepted 18 May 2012

\section{ABSTRACT}

Aims. There is some controversy on the nature of dust clouds found in direction of the interacting galaxy triplett M 81, M 82, and NGC 3077. Are they associated with the tidal arms seen in HI around those galaxies or are they simply Galactic foreground clouds? Methods. Data from the SPIRE instrument onboard Herschel ${ }^{\star \star}$ and MIPS onboard of Spitzer are used to derive physical parameters for the dust clouds. These observions are compared to CO clouds previously mapped with the IRAM and the FCRAO radio telescopes. Results. SPIRE and MIPS maps show several dust clouds north of M 81 and south of NGC 3077. Modelling of the dust emission provides total hydrogen column densities between 1.5 and $5.0 \times 10^{20} \mathrm{~cm}^{-2}$. Dust temperatures are between 13 to $17 \mathrm{~K}$. No significant difference in the dust emission can be found between individual clouds. It is shown that $\mathrm{CO}$ line emission provides the best clues on the origin of those clouds. Most of the clouds seen towards M 81 are associated with small-area molecular structures (SAMS), i.e. tiny CO clouds of Galactic origin. The clouds seen towards NGC 3077 are partly associated with the tidal arms and are partly in the Galactic foreground associated with SAMS
\end{abstract}

Key words. ISM: clouds - ISM: individual objects: SAMS1 - ISM: individual objects: SAMS2 - galaxies: individual: M 81 ISM: molecules - galaxies: individual: NGC 3077

\section{Introduction}

Interpretation of astronomical observations is always hampered by the lack of direct distance information. Whether or not objects on the same line-of-sight are indeed physically related is not easy to judge. Directly linked to this question is the problem of finding dust in the intergalactic medium (Xilouris et al. 2006; Walter et al. 2011). One well studied region where this problem becomes obvious is the region towards M 81, M 82, and NGC 3077. It is one of the closest sample of interacting galaxies. HI tidal arms are found to connect these three galaxies (Yun et al. 1994). Especially since the discovery of Arp's loop (Arp 1965 ) there has been some debate about the nature of interstellar clouds in that region: are they related to tidal arms around the interacting galaxy triplett (Sun et al. 2005; de Mello et al. 2008) or to Galactic foreground cirrus (Sollima et al. 2010; Davies et al. 2010)?

Sandage (1976) presented deep optical images that showed that the M 81-M 82-NGC 3077 region is seen through wide spread Galactic foreground cirrus clouds. De Vries et al. (1987) presented large-scale maps with an angular resolution of 9 arcmin of atomic hydrogen, carbon monoxide and dust infrared emission, which showed Galactic cirrus emission towards the M 81 triple with total hydrogen column densities of about $1-2 \times 10^{20} \mathrm{~cm}^{-2}$. In most cases it is possible to distinguish between an extragalactic or Galactic origin of the dust and gas because the radial velocities are very different from each other.

\footnotetext{
* New address: Institut für Physik und ihre Didaktik, Universität zu Köln, Gronewaldstr. 2, 50931 Köln, Germany.

$\star \star$ Herschel is an ESA space observatory with science instruments provided by European-led Principal Investigator consortia and with important participation from NASA.
}

In the case of the M81 region however the radial velocities of Galactic and extragalactic gas share (at least partly) the same local standard of rest (LSR) velocity range close to zero.

Significant substructure in the Galactic foreground gas was detected by Heithausen (2002) who found several small-area molecular structures (SAMS) towards the outer regions of M 81 and NGC 3077. SAMS are tiny molecular clouds detected in a region where the shielding of the interstellar radiation field is too low for them to survive for a long time (Heithausen 2002). The CO clouds are less than one arcmin wide, corresponding to linear sizes below $6000 \mathrm{AU}$ at an adopted distance of $100 \mathrm{pc}$. High angular resolution observations obtained with the Plateau de Bure interferometer show substructure down to the resolution limit of $3^{\prime \prime}$ (approx. $300 \mathrm{AU}$ ) with indication of further unresolved structure (Heithausen 2004).

So far, one such cloud has been detected towards the tidal arms around NGC 3077 and four in the region around M 81 (Heithausen 2006). The agreement of the LSR velocities of SAMS with that of the Galactic HI gas shows that they are of Galactic origin. Furthermore their CO linewidths are too narrow for extragalactic objects. At an adopted distance of about $100 \mathrm{pc}$ their masses are about that of Jupiter.

The purpose of this paper is twofold: first independent estimates on column densities and temperatures of SAMSs are obtained, second these objects are used to distinguish between Galactic foreground clouds and extragalactic tidal arm clouds. The outline of the paper is as follows: after a brief description of the infrared data the resulting spectral energy distributions (SEDs) are used to determine dust column densities and temperatures and find new SAMS candidates; one of those could directly be confirmed as molecular cirrus cloud with existing $\mathrm{CO}$ data. Based on published $\mathrm{CO}$ data the dust clouds are 


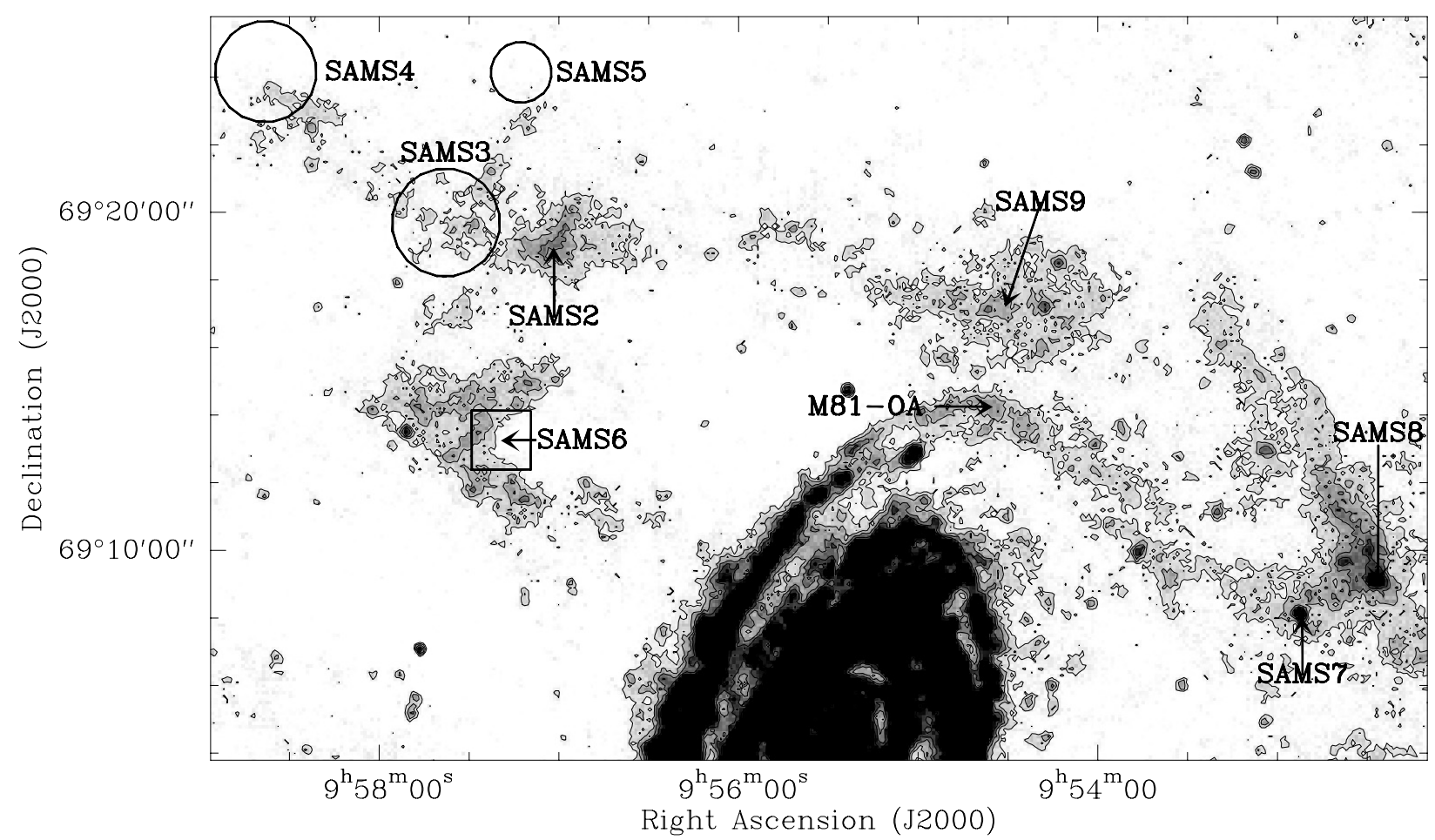

Fig. 1. SPIRE $250 \mu \mathrm{m}$ map of the area surrounding SAMS2. Contours are from 0.02 to $0.1 \mathrm{Jy}^{\text {beam }}{ }^{-1}$ every $0.02 \mathrm{Jy}$ beam $^{-1}$. The intense emission at the lower centre of the map originates from M 81. The positions of the sources discussed in this paper are labelled. The weak clouds SAMS 3-5 are marked by circles with the approximate size of the clouds. The square marks the area of SAMS6 over which spectra have been averaged to detect a weak CO line (see Fig. 4).

classified as Galactic or extragalactic. It is shown that the dust clouds towards M 81 are most likely Galactic foreground clouds, whereas the clouds seen towards NGC 3077 are partly associated with the tidal arms and are partly in the Galactic foreground.

\section{Observations}

For the analysis in this Paper I used photometric data obtained with the SPIRE (Griffin et al. 2010) oboard of the Herschel satellite and with MIPS (Rieke et al. 2004) onboard of the Spitzer satellite. Calibrated data of the M 81 region obtained with SPIRE were retrieved from the Herschel archive (level 2 products). A first analysis of the data is presented by Bendo et al. (2010) to study the dust content and temperature of the spiral galaxy. SPIRE data of NGC 3077 were obtained from the open time key project KINGFISH (Walter et al. 2011).

The SPIRE data are given in $\mathrm{Jy} \mathrm{beam}^{-1}$; to convert the data to $\mathrm{MJy} \mathrm{sr}{ }^{-1}$ beam areas of 447,816 , and $1711 \mathrm{arcsec}^{-1}$ were assumed for the 250, 350, and $500 \mu \mathrm{m}$ bands (Sibthorpe et al. 2011). The calibration uncertainties for the individual bands are $7 \%$ (SPIRE Observor's Manual 2011). At 250, 350 and $500 \mu \mathrm{m}$ SPIRE has a slightly elliptical beam of $18.7^{\prime \prime} \times 17.5^{\prime \prime}, 25.6^{\prime \prime} \times$ $24.2^{\prime \prime}$ and $38.2^{\prime \prime} \times 34.6^{\prime \prime}$, respectively (Sipthorpe et al. 2011, loc.cit.) for pixel scales of 6,10 , and 14 arcsec.

For the M 81 region photometric data at 70 and $160 \mu \mathrm{m}$ obtained with Spitzer MIPS were retrieved from the SINGS archive (Kennicutt et al. 2003). Corresponding data at $160 \mu \mathrm{m}$ for the region around NGC 3077 were taken directly from the Spitzer archive. For this region three data sets exist in the archive, which cover the clouds of interest completely, though part of the more extensive diffuse emission around the galaxy might be missing. Because the signal-to-noise level of a single set is already high enough (larger than 10 for the weakest cloud) I only used one data set (AOR 17597952), after checking that the data set is comparable with the other sets (AORs 17598208 and 17597696). The data sets are calibrated in MJy/sr. The FWHM of the pointspread function (PSF) is $18^{\prime \prime}$, and $40^{\prime \prime}$ at $70 \mu \mathrm{m}$ and $160 \mu \mathrm{m}$, resp. (Engelbracht et al. 2004). The accuracy of the photometric calibration of the two bands was estimated to be $5 \%$ at $70 \mu \mathrm{m}$ (Gordon et al. 2007) and 12\% at $160 \mu \mathrm{m}$ (Stansberry et al. 2007).

Additionally to the photometric observations, the IRAM $30 \mathrm{~m}$ telescope has been used to search for extensive ${ }^{12} \mathrm{CO}(1 \rightarrow 0)$ emission around SAMS1 in June 2001. Observations were done with a wobbling subreflector with an offposition separated by $200^{\prime \prime}$ in azimuth from the on-position. An autocorrelator spectrometer was used with a velocity resolution of $0.2 \mathrm{~km} \mathrm{~s}^{-1}$. The angular resolution of the telesccope at $115 \mathrm{GHz}$ was $22^{\prime \prime}$. The data were processed using the standard data reduction software for radioastronomical spectra GILDAS ${ }^{1}$. Only linear baselines were removed from the spectra.

\section{Results}

\subsection{The region towards $M 81$}

The SPIRE $250 \mu \mathrm{m}$ map of the M 81 region is presented in Fig. 1. Dust is concentrated in several small filaments. Six clouds are identified outside the main body of M 81. Positions of the local maxima are listed in Table 1.

For comparison the distribution of the total HI gas of this region taken from the THINGS project (Walter et al. 2008) is displayed in Fig. 2. The velocity range of the naturally weighted

$\overline{1}$ see http://www.iram.fr/IRAMFR/GILDAS/ 


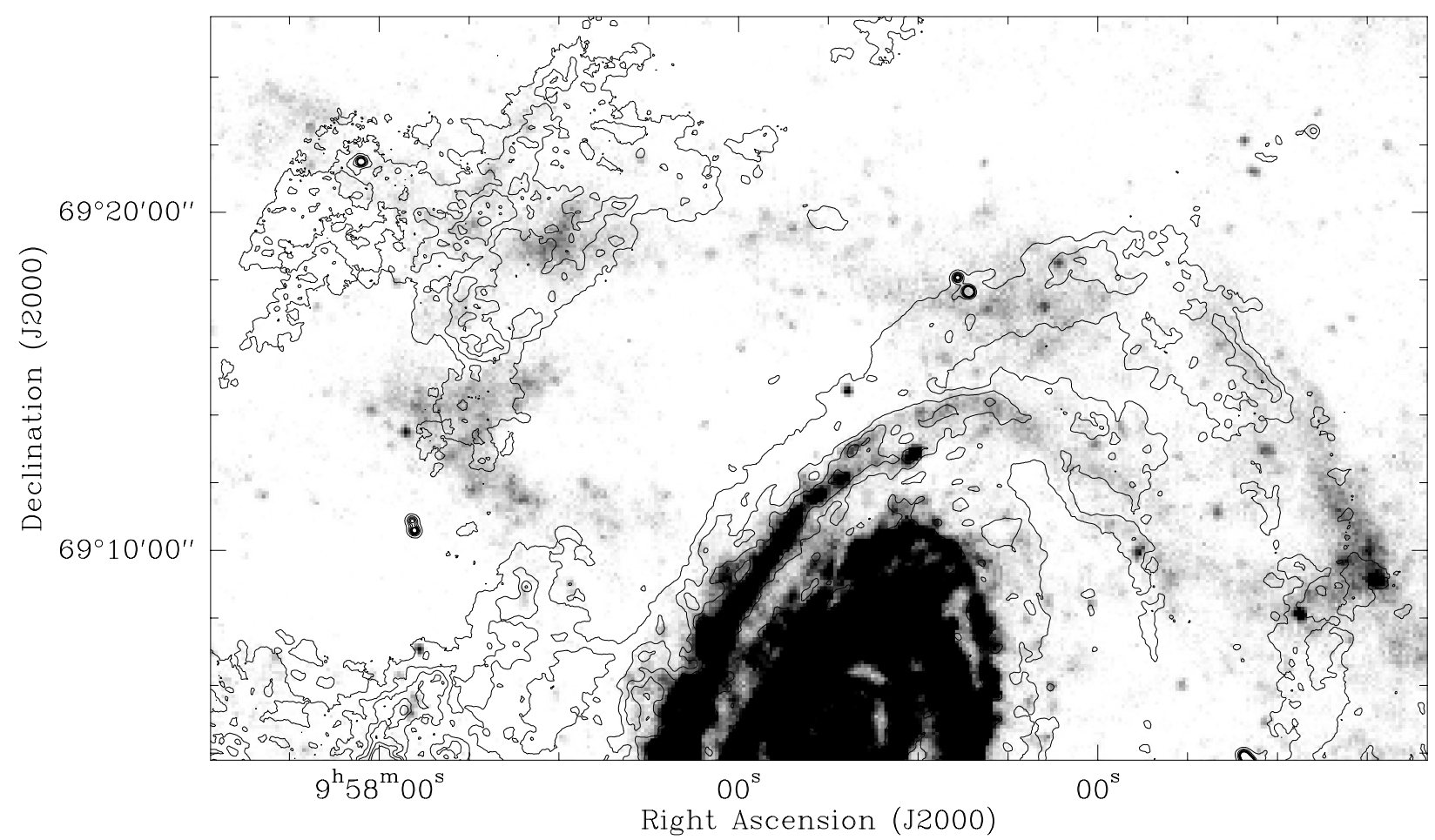

Fig. 2. THINGS total HI map (contours, Walter et al. 2008) of the area surrounding M 81 overlayed on the SPIRE $250 \mu \mathrm{m}$ map (greyscale). The map has been integrated over a velocity range of about $250 \mathrm{~km} \mathrm{~s}^{-1}$. Contours are from 50 to $500 \mathrm{Jy} \mathrm{beam}^{-1} \mathrm{~m} \mathrm{~s}^{-1}$ every $100 \mathrm{Jy} \mathrm{beam}^{-1} \mathrm{~m} \mathrm{~s}^{-1}$.

Table 1. Parameters of the dust clouds derived from SPIRE and MIPS data.

\begin{tabular}{lccccccccc}
\hline \hline Source & $\begin{array}{c}\text { RA } \\
\text { J2000 }\end{array}$ & $\begin{array}{c}\text { Dec } \\
\mathrm{J} 2000\end{array}$ & $\begin{array}{c}I_{250 \mu \mathrm{m}} \\
\mathrm{MJy} \mathrm{sr}^{-1} \\
\text { SPIRE }\end{array}$ & $\begin{array}{c}I_{350 \mu \mathrm{m}} \\
\mathrm{MJy} \mathrm{sr}^{-1} \\
\text { SPIRE }\end{array}$ & $\begin{array}{c}I_{500 \mu \mathrm{m}} \\
\mathrm{MJy} \mathrm{s}^{-1} \\
\text { SPIRE }\end{array}$ & $\begin{array}{c}I_{70 \mu \mathrm{m}} \\
\mathrm{MJy} \mathrm{sr}^{-1} \\
\mathrm{MIPS}\end{array}$ & $\begin{array}{c}I_{160 \mu \mathrm{m}} \\
\mathrm{MJy} \mathrm{sr}^{-1} \\
\mathrm{MIPS}\end{array}$ & $\begin{array}{c}T_{\text {Dust }} \\
\mathrm{K}\end{array}$ & $\begin{array}{c}N_{\mathrm{H}} \\
10^{20} \mathrm{~cm}^{-2}\end{array}$ \\
\hline SAMS1 & $10: 03: 28.4$ & $68: 41: 29.2$ & 3.9 & 2.1 & 1.0 & $\ldots$ & 4.5 & 14.5 & 1.8 \\
NGC 3077-TA1 & $10: 04: 00.6$ & $68: 41: 09.9$ & 5.7 & 3.6 & 2.0 & $\ldots$ & 4.9 & 13 & 5.4 \\
NGC 3077-TA2 & $10: 03: 55.4$ & $68: 42: 39.4$ & 4.2 & 2.7 & 1.5 & $\ldots$ & 5.1 & 13 & 3.5 \\
NGC 3077-TA3 & $10: 03: 48.0$ & $68: 42: 01.4$ & 4.7 & 2.8 & 1.6 & $\ldots$ & 5.1 & 13 & 3.6 \\
SAMS2 & $9: 57: 01.0$ & $69: 19: 05.4$ & 7.6 & 3.5 & 1.5 & $\leq 0.5$ & 7.4 & 16 & 3.3 \\
SAMS3 & $9: 57: 36.8$ & $69: 19: 48.7$ & 3.6 & $\ldots$ & $\ldots$ & $\ldots$ & $\ldots$ & $\ldots$ & $\ldots$ \\
SAMS4 & $9: 58: 36.5$ & $69: 24: 16.8$ & 4.6 & 2.3 & 0.9 & $\ldots$ & $\ldots$ & 13.5 & 3.7 \\
SAMS5 & $9: 57: 11.9$ & $69: 24: 15.3$ & 2.7 & $\ldots$ & $\ldots$ & $\ldots$ & $\ldots$ & $\ldots$ & $\ldots$ \\
SAMS6 & $9: 57: 18.5$ & $69: 13: 22.4$ & 5.7 & 3.0 & 1.5 & $\leq 0.5$ & 6.7 & 15.5 & 3.1 \\
SAMS7 & $9: 52: 53.1$ & $69: 08: 09.6$ & 8.6 & 3.8 & 1.5 & 2.9 & 8.0 & 16.5 & 3.1 \\
SAMS8 & $9: 52: 28.0$ & $69: 09: 06.8$ & 11.2 & 5.2 & 1.9 & $\leq 0.5$ & 10.9 & 14.5 & 7.2 \\
SAMS9 & $9: 54: 31.4$ & $69: 17: 19.5$ & 6.3 & 3.4 & 1.6 & $\leq 0.5$ & 5.4 & 13 & 6.9 \\
M 81-OA & $9: 54: 35.5$ & $69: 14: 22.0$ & 6.5 & 3.6 & 1.3 & $\leq 0.5$ & 5.5 & 14 & 5.1 \\
\hline
\end{tabular}

Notes. 1: possible contributions from a point source.

maps covers about $250 \mathrm{~km} \mathrm{~s}^{-1}$. Note that because the interferometer data are not corrected for missing zero spacing extensive HI emission has been filtered out. Due to the small size of the dust clouds this is however not critical for the analysis. The distribution of $\mathrm{HI}$ associated with the spiral arms of M 81 shows a close similarity to the dust. The HI clouds associated with the tidal arms of M 81 show however no obvious morphological similarity to the dust clouds. Davies et al. (2010) already noted that a much greater similarity can be found when comparing the infrared data with a single velocity channel of the HI data at velocities close to zero, i.e. that which correspond to Galactic velocities.

A morphological similarity can be found when one compares the dust with the CO emission from SAMS, too. SAMS2 (Heithausen 2002) can clearly be identified in all SPIRE bands (compare Figs. 1 and 3) and in the MIPS $160 \mu \mathrm{m}$ band. The other molecular structures in its vicinity found in $\mathrm{CO}$ observations with the FCRAO $14 \mathrm{~m}$ telescope (Heithausen 2006) are detected in the $250 \mu \mathrm{m}$ map, too. Note that the CO data have a coarser angular resolution of only $45^{\prime \prime}$ compared to the SPIRE $250 \mu \mathrm{m}$ data and are very weak $\left(T_{\mathrm{A}}^{*}<0.1 \mathrm{~K}\right)$.

There is one further dust cloud in the FCRAO field (compare Figs. 1 and 3) previously undetected in CO (labelled SAMS6 in Fig. 1). To see whether molecular gas is associated with that cloud the spectra obtained with the FCRAO $14 \mathrm{~m}$ telescope (Heithausen 2006) were averaged over areas of $60^{\prime \prime}$ by $60^{\prime \prime}$. In those spectra four neighboring positions with marginal $\mathrm{CO}$ detections can be found centered on the Galactic coordinates $(l, b)=\left(141^{\circ} .802,40.936\right)$ which corresponds to $(\alpha, \delta)=\left(09^{\mathrm{h}} 57^{\mathrm{m}} 18^{\mathrm{s}} .5,+69^{\circ} 13^{\prime} 22^{\prime \prime}\right.$. 4$)$. The average of 


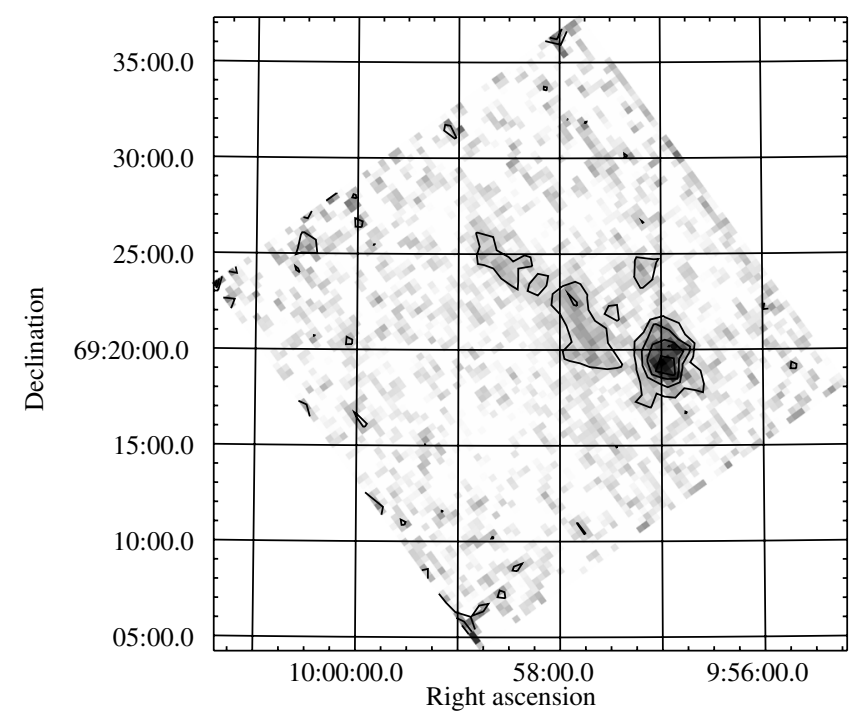

Fig. 3. Integrated CO map obtained with the FCRAO $14 \mathrm{~m}$ radiotelescope (Heithausen 2006). Contours are every $0.04 \mathrm{~K} \mathrm{~km} \mathrm{~s}^{-1}$ starting at $0.04 \mathrm{~K} \mathrm{~km} \mathrm{~s}^{-1}$.

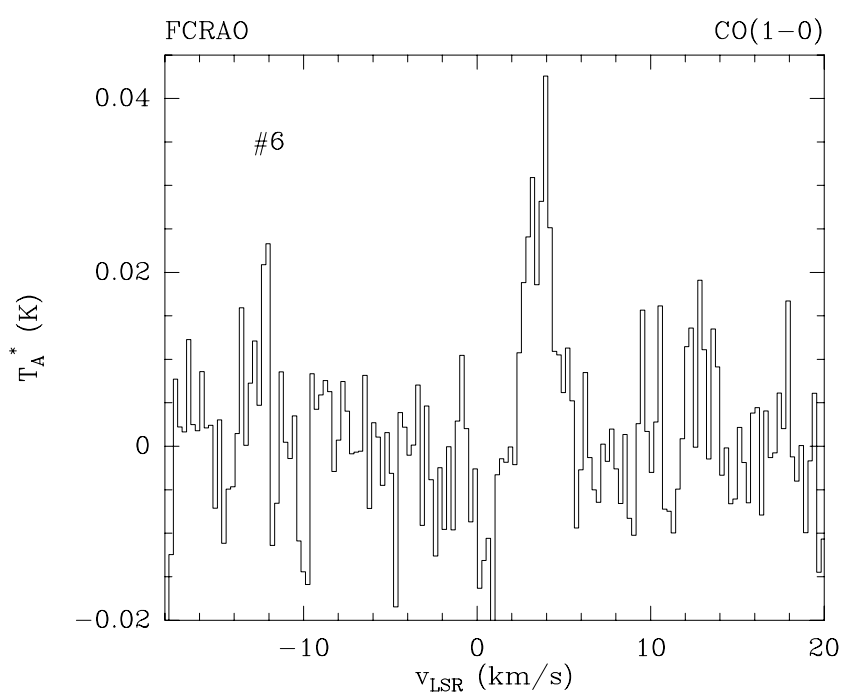

Fig. 4. $\mathrm{CO}(1 \rightarrow 0)$ spectrum of the newly detected SAMS6 centered on $(\alpha, \delta)=\left(09^{\mathrm{h}} 57^{\mathrm{m}} 18.5,+69^{\circ} 13^{\prime} 22^{\prime \prime} \cdot 4\right)$.

those positions is shown in Fig. 4. Here a clear CO line has been detected with the following values obtained from a Gaussian fit to the data: amplitude $T_{\mathrm{A}}^{*}=0.032 \pm 0.008 \mathrm{~K}$, center velocity $v_{\text {LSR }}=3.6 \pm 0.1 \mathrm{~km} \mathrm{~s}^{-1}$, and line width $\Delta v=1.8 \pm 0.3 \mathrm{~km} \mathrm{~s}^{-1}$. These values are similar to those of previous detected SAMS.

For the further quantitative analysis (Sect. 3.3) six apparent cirrus clouds are identified, three of which show $\mathrm{CO}$ emission of clear Galactic origin (SAMS2, 4, and 6) while for the other three such observations are not yet available (labelled SAMS7, 8, and 9 in Table 1 and Fig. 1). Although SAMS3 and SAMS5 show $\mathrm{CO}$ emission, they are not considered further because they are hard to identify in the SPIRE 350 and $500 \mu \mathrm{m}$ bands. For comparison one cloud in an outer spiral arm of M 81 (labelled M 81-OA is also listed in Table 1).

\subsection{The region towards NGC 3077}

NGC 3077 is the smallest member of the interacting M 81 galaxy triple. Intense HI tidal arms have been found surrounding

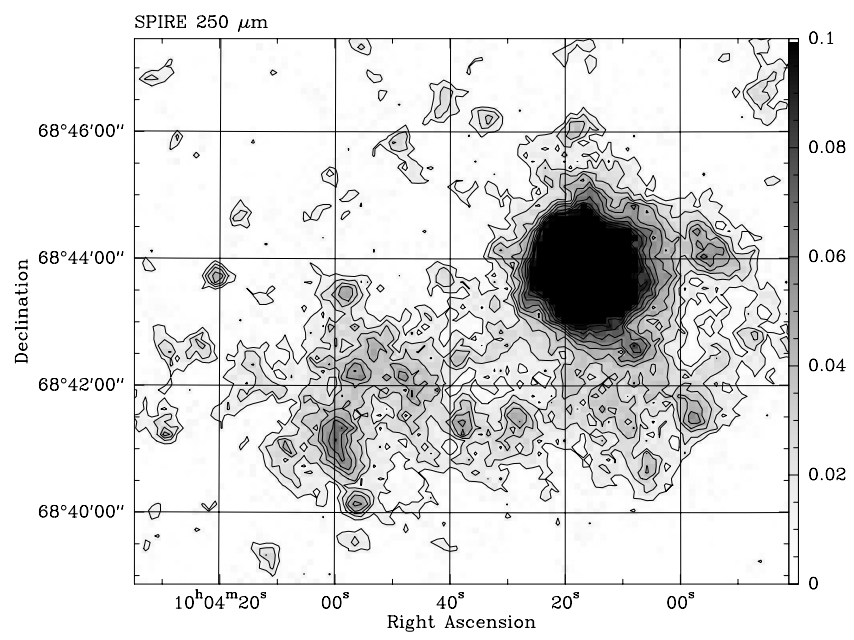

Fig. 5. Spire $250 \mu \mathrm{m}$ map of the region towards NGC 3077 as observed by Walter et al. (2011). Contours are from 0.01 to $0.08 \mathrm{Jy}^{-1}$ beam ${ }^{-1}$ every $0.01 \mathrm{Jy} \mathrm{beam}^{-1}$. The intense emission in the right half of the map originates from NGC 3077.

this galaxy (Yun et al. 1994). Towards these arms Walter \& Heithausen (1999) have detected a giant molecular complex. Walter et al. (2011) have used the SPIRE instrument to search for the thermal emission of dust asociated with the tidal arms. A map at a wavelength of $250 \mu \mathrm{m}$ is presented in Fig. 5. Based on a positional coincidence Walter et al. attribute all of the emission they found in that region to the tidal arms around NGC 3077. In this section it is shown that at least some part is most likely associated with Galactic foreground clouds.

In the region towards NGC 3077 we are faced with two molecular clouds, one of clear extragalactic and one of clear Galactic origin. The situation is displayed in Fig. 6. The distinction can be made based on the CO linewidth and LSR velocity. The extragalactic cloud complex (Walter \& Heithausen 1999; Heithausen \& Walter 2000) shows CO linewidths of several $\mathrm{km} \mathrm{s}^{-1}$ and velocities slightly outside that of the local Galactic gas (solid contours). Next to this complex is the small-area molecular structure SAMS1 (dashed contours, Heithausen 2002) with a linewidth of below $1 \mathrm{~km} \mathrm{~s}^{-1}$ and LSR velocities in agreement with that of local Galactic HI gas.

Beyond the CO map of SAMS1 presented in Fig. 6 further $\mathrm{CO}(1 \rightarrow 0)$ emission has been detected with the IRAM $30 \mathrm{~m}$ telescope towards the position $(\alpha, \delta)=\left(10^{\mathrm{h}} 03^{\mathrm{m}} 08^{\mathrm{s}} .4\right.$, $+68^{\circ} 40^{\prime} 31^{\prime \prime} 8$ ). A Gaussian fit to the data gives a amplitude $T_{\mathrm{A}}^{*}=$ $0.055 \pm 0.004 \mathrm{~K}$, a center velocity $v_{\mathrm{LSR}}=3.47 \pm 0.14 \mathrm{~km} \mathrm{~s}^{-1}$, and a line width $\Delta v=1.6 \pm 0.3 \mathrm{~km} \mathrm{~s}^{-1}$. (The LSR velocity corresponds to a heliocentric velocities of $v_{\mathrm{Hel}}=-2.53 \mathrm{~km} \mathrm{~s}^{-1}$.) These values are similar to that of SAMS1, i.e. the molecular gas is of Galactic origin.

Both the Galactic and the extragalactic clouds are associated with emission in the $250 \mu \mathrm{m}$ band (see Fig. 6) as well as with emission in the 350 and $500 \mu \mathrm{m}$ band. For further quantitative analysis three extragalactic regions (NGC 3077-TA1 to TA3) and one Galactic cloud (SAMS1) are identified (see Table 1). These positions are associated with the most intense regions in this SPIRE $250 \mu \mathrm{m}$ map outside the main body of NGC 3077.

\subsection{SEDS}

To obtain dust column densities and temperatures the IR maps were smoothed to the same angular resolution and intensities 


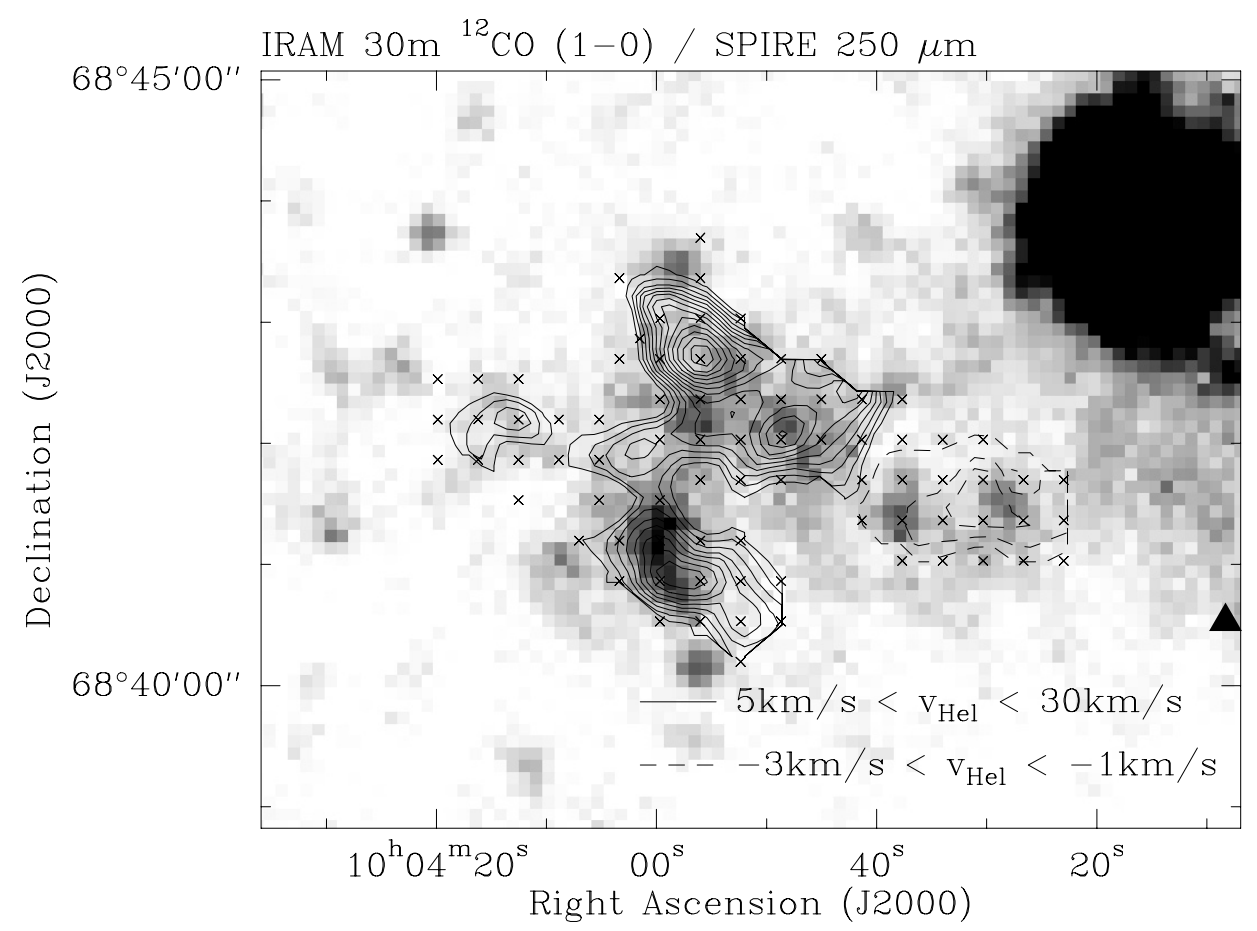

Fig. 6. Spire $250 \mu \mathrm{m}$ map of the region towards NGC 3077 overlaid with contours from observations of the $\mathrm{CO}(1 \rightarrow 0)$ line obtained with the IRAM $30 \mathrm{~m}$ telescope (Heithausen 2002; Walter \& Heithausen 1999; Heithausen \& Walter 2000). Observed CO positions are marked by Xs. Contours are from $0.1 \mathrm{~K} \mathrm{~km} \mathrm{~s}^{-1}$ every $0.1 \mathrm{~K} \mathrm{~km} \mathrm{~s}^{-1}$. Solid contours show emisson from the tidal arm cloud associated with NGC3077 (Walter \& Heithausen 1999; Heithausen \& Walter 2000), whereas dashed contours show emission from the Galactic small area molecular structure SAMS1 (Heithausen 2002). There is further $\mathrm{CO}$ emission towards the position $(\alpha, \delta)=\left(10^{\mathrm{h}} 03^{\mathrm{m}} 08 \mathrm{~s} .4\right.$, $+68^{\circ} 40^{\prime} 31^{\prime \prime} 8$ ), which is marked by the black triangle. averaged over square areas with $40^{\prime \prime}$ width were obtained. Background correction were obtained in fields close to the observed regions which show no obvious emission. Derived intensities for the individual spectral bands are listed in Table 1. The uncertainties for the values are estimated from the noise level $(1 \sigma)$ in regions, which are apparently free of emission. For the $\mathrm{M} 81$ region these values are found to be at $0.60,0.38$ and $0.21 \mathrm{MJy} \mathrm{sr}^{-1}$ for the Herschel 250,350, and $500 \mu \mathrm{m}$ bands and $0.5 \mathrm{MJy} \mathrm{sr}^{-1}$ for both the MIPS 70 and the $160 \mu \mathrm{m}$ bands. For the NGC 3077 region the correspoinding values are $0.55,0.27$ and $0.16 \mathrm{MJy} \mathrm{sr}^{-1}$ for the Herschel 250,350, and $500 \mu \mathrm{m}$ bands and $0.5 \mathrm{MJy} \mathrm{sr}^{-1}$ for the MIPS $160 \mu \mathrm{m}$ band, respectively.

A blackbody fit is used to derive dust temperatures and hydrogen column densities of the observed regions. Assuming optically thin emission the observed intensity $I_{v}$ can be modelled as

$$
I_{v}=B_{v} m_{\mathrm{H}} \mu N_{\mathrm{H}} \kappa_{v}
$$

where $B_{v}$ is the Planck function and $N_{\mathrm{H}}$ is the total hydrogen column density (cf. Ward-Thompson et al. 2010). $m_{\mathrm{H}} \mu$ is the mean mass of a particle in the cloud with $m_{\mathrm{H}}$ being the mass of an hydrogen atom; $\mu$ was adopted to be 2.3 (cf. Ade et al. 2011). Following Beckwith et al. (1990) the dust mass opacity $\kappa_{v}$ is parameterized as

$$
\kappa_{v}=0.1 \mathrm{~cm}^{2} \mathrm{~g}^{-1}\left(\frac{v}{1000 \mathrm{GHz}}\right)^{\beta} .
$$

For the fit a dust opacity index $\beta=2$ has been adopted, which is consistent with the value used by André et al. (1993) for molecular cores. Fits to the data are displayed in Fig. 7; derived temperatures and total hydrogen column densities are listed in Table 1.

The derived dust temperatures are within the range of 13 to $17 \mathrm{~K}$ with an uncertainty of $\Delta T= \pm 2 \mathrm{~K}$ for individual values. There is no significant difference between the dust temperature of the outer arm of M 81, the tidal arm features close to NGC 3077, and the Galactic foreground clouds. The values are similar to those derived for typical cirrus clouds as e.g. the Polaris Flare where the average dust temperature was found to be $T_{\mathrm{D}}=14.5 \pm 1.6 \mathrm{~K}$ (Miville-Deschênes et al. 2010). These values agree also well with those estimated from exciation conditions of the CO line between 7 to $20 \mathrm{~K}$ (Heithausen 2004), although gas and dust are not necessarily coupled.

The total hydrogen column densities estimated from the SED fit are within 1.8 and $7.2 \times 10^{20} \mathrm{~cm}^{-2}$. The uncertainty is estimated to be $\pm 0.5 \times 10^{20} \mathrm{~cm}^{-2}$. Again no significant difference between extragalactic and Galactic clouds is apparent. For SAMS1 Heithausen (2002) found an HI column density of $N(\mathrm{HI})=1.3 \times 10^{20} \mathrm{~cm}^{-2}$ and an $\mathrm{H}_{2}$ column densities of $N\left(\mathrm{H}_{2}\right)=$ $0.7 \times 10^{19} \mathrm{~cm}^{-2}$ and for SAMS2 N(HI) $=2.1 \times 10^{20} \mathrm{~cm}^{-2}$ and $N\left(\mathrm{H}_{2}\right)=3.7 \times 10^{19} \mathrm{~cm}^{-2}$. The sums of $N(\mathrm{H})=N(\mathrm{HI})+2 \times N\left(\mathrm{H}_{2}\right)$ agree well with the values derived here from the IR emssion for both clouds.

\section{Discussion}

\subsection{SAMS}

Previous estimates on the kinetic temperatures and masses of SAMSs (Heithausen 2002, 2006) were made using HI and $\mathrm{CO}$ data assuming that the physical parameters are similar to those of Galactic cirrus clouds. While the determination of the atomic hydrogen column density is straightforward from its $21 \mathrm{~cm}$ line, the determination of the molecular hydrogen column density is more difficult. It is based on an empirically determined conversion factor $X_{\mathrm{CO}}$ applied to the velocity integrated CO line. For SAMS the low value found for the large scale foreground clouds (de Vries et al. 1987) had been used.

SPIRE and MIPS data now provide an independent means to estimate the dust temperatures and masses of SAMSs. In this paper the values were derived adopting a gas-to-dust mass ratio of 100, which is well within the values found for our Galaxy (Sodroski et al. 1997). These new estimates confirm the previous ones, namely that SAMSs have low total hydrogen column densities and, following from that, low masses, comparable to that of Jupiter. Their temperatures are between 13 and $17 \mathrm{~K}$. SAMSs therefore form most likely the very low mass end of molecular 


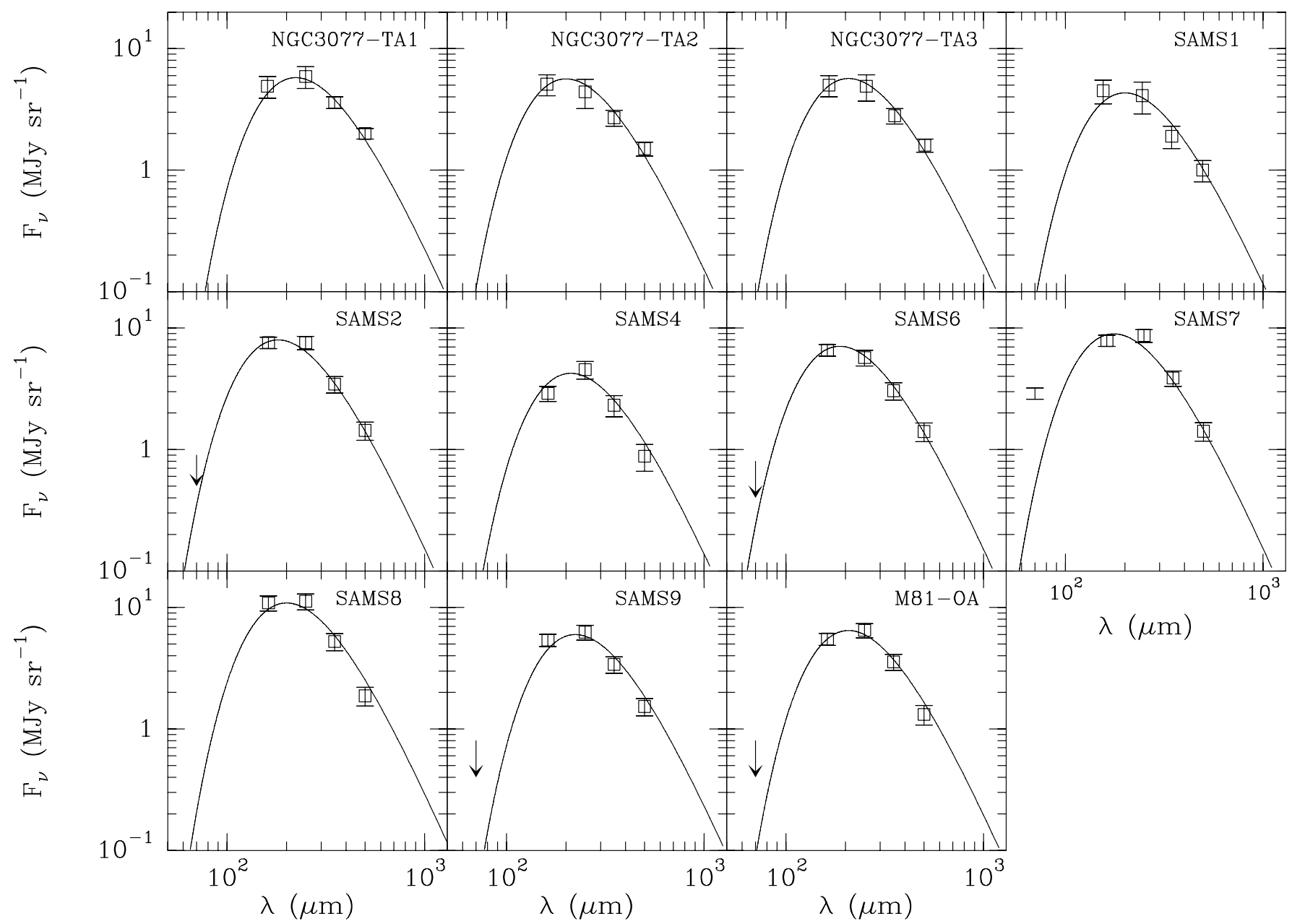

Fig. 7. Dust spectra of the observed sources obtained from the SPIRE and MIPS bands. Each point gives the mean intensity of a field covering an area of $40^{\prime \prime} \times 40^{\prime \prime}$. Solid lines are fits to the data. For the fits $\beta=2$ has been adopted. The $70 \mu \mathrm{m}$ intensity of SAMS7 shows contributions from a point source.

clouds in the Galactic cirrus. As such they can provide valuable clues for the understanding of the formation or destruction of molecular clouds in our Galaxy.

Note that this set of observations however does not provide independent clues on the metallicty of the clouds for the following reason: the $X_{\mathrm{CO}}$ factor is found to increase with decreasing metallicity (e.g. Wilson 1995; Barone et al. 2000). For a given $\mathrm{CO}$ intensity a lower metallicity would therefore result in a higher $\mathrm{H}_{2}$ column density. Similarly, the gas-to-dust ratio increases with decreasing metallicity. For a lower metallicity a given infrared intensity would thus result in a higher $\mathrm{H}_{2}$ column density, too.

\subsection{Tidal arms or Galactic cirrus clouds?}

The dust temperatures and column densities of the clouds examined in this paper are very similar. Based on the assumption of a Galactic dust-to-gas ratio total hydrogen column densities are found to be in the range between 1.8 and $7.2 \times 10^{20} \mathrm{~cm}^{-2}$. Dust temperatures are between 13 to $17 \mathrm{~K}$. There is no obvious difference between the individual clouds. So there is no way to distinguish between Galactic and extragalactic origin based on dust colour ratios or infrared intensity alone.

In the region towards M 81 at least three out of six clouds are clearly detected in CO lines of Galactic origin. The other filamentary clouds have similar structures and similar intensities in the SPIRE bands. They are thus candidates for SAMS, which have however to be verified by deep CO observations.

Comparison of deep optical images (e.g. given in Sollima et al. 2010) with the SPIRE $250 \mu \mathrm{m}$ map shows that the clouds detected in CO (SAMS2, 4, and 6) are morphological very similar. These dust clouds form part of the structure known as Arp's ring or loop (Arp 1965). The narrow CO linewidth and the close agreement of the line of sight velocities with local HI gas (Heithausen 2002, 2004) clearly rule out an extragalactic origin of this structure. We are thus just faced with a chance superposition of foreground filaments forming a loop like structure with the distant galaxy M 81. The observations presented in this paper thus confirm the conclusions of Sollima et al. (2010) who, based on $\mathrm{HI}$ and dust observations, found that the bulk of dust emission outside the main body of M 81 is associated with Galactic cirrus clouds. Davies et al. (2010) describe a break-down of the HI-infrared relation on scales below 1 arcmin. This could be caused by the fact that the clouds become at least partly molecular, as can be seen by the detection of $\mathrm{CO}$ towards some of these clouds.

Towards NGC 3077 the situation is more complex: here we are faced with one molecular cloud of Galactic and another molecular complex of extragalactic origin. Both show up in all SPIRE bands with similar colour ratios. Our incomplete CO map presented in this paper shows that the Galactic cloud SAMS1 is 
probably more extended than previously thought. This suggests that even more of the dust seen towards NGC 3077 is located in the Galactic foreground. Walter et al. (2011) attributed all SPIRE emission to the tidal tail of NGC 3077, whereas it is shown here that some of the IR emission is of Galactic origin.

\section{Conclusions}

The data presented in this paper show that the mere coincidence of HI gas and dust emission on the same line of sight does not proof that the clouds are really physically associated, even if the intensity maxima partly coincide. $\mathrm{CO}$ gas is a much better indicator for an association. The velocity information of the $\mathrm{CO}$ spectral lines provides valuable clues on the origin of the gas and the associated dust, either Galactic or extragalactic. To confirm or exclude either origin, $\mathrm{CO}$ spectra should therefore cover a bandwidth which includes both Galactic and extragalactic velocities. Because in some cases such a configuration is not possible with the receiver, the observer should consider to take at least some control spectra covering Galactic velocities.

Clearly, more extensive CO observations of the Galactic cirrus clouds are demanded to study the importance of small area molecular structures. Currently most of such surveys for molecular cirrus clouds are done with moderate to low angular resolution (Heithausen et al. 1993; Magnani et al. 2000; Onishi et al. 2001). Due to the small size of SAMSs these observations should be conducted at high angular resolution and high sensitivity. As shown in this paper emission at 250 to $500 \mu \mathrm{m}$ could be a good guide to find such clouds.

Finally, it would be interesting to study if there are more examples of dust clouds where their origin, either Galactic or extragalactic, is not clear. Two such cases have been reported by Dirsch et al. (2003, 2005) and by Cortese et al. (2010). Dirsch et al. found a single dense cloud of only 4 " diameter projected on a spiral arm of the galaxy NGC 3269. Based on a study of the redding law of that object the authors conclude that it has a Galactic origin. Cortese et al. describe a system of interacting galaxies similar to that of M 81. Towards NGC 4435/4438 they found a $\mathrm{HI}, \mathrm{CO}$, and dust cloud which could be interpreted as tidal stream or as Galactic foreground cirrus. Based on velocity information of $\mathrm{HI}$ and $\mathrm{CO}$ spectra the authors favour a Galactic origin. The width of their CO line of only $\Delta v=1.5 \mathrm{~km} \mathrm{~s}^{-1}$ is similar to that of SAMS (Heithausen 2006), i.e. more consistent with Galactic cirrus clouds than with extragalactic molecular complexes. It is certainly worthwhile to map the total extend of the molecular cloud in the NGC 4435/4438 field and to search CO towards NGC 3269, because they could be similar to the SAMSs described in this paper.
Acknowledgements. I thank Fabian Walter for critical comments on the manuscript. SPIRE has been developed by a consortium of institutes led by Cardiff University (UK) and including Univ. Lethbridge (Canada); NAOC (China); CEA, LAM (France); IFSI, Univ. Padua (Italy); IAC (Spain); Stockholm Observatory (Sweden); Imperial College London, RAL, UCL-MSSL, UKATC, Univ. Sussex (UK); and Caltech, JPL, NHSC, Univ. Colorado (USA). This development has been supported by national funding agencies: CSA (Canada); NAOC (China); CEA, CNES, CNRS (France); ASI (Italy); MCINN (Spain); SNSB (Sweden); STFC (UK); and NASA (USA).

\section{References}

Ade, P. A. R., Aghanim, N., Arnaud, M., et al. 2011, A\&A, 536, A22 André, P., Ward-Thompson, D., \& Barsony, M. 1993, ApJ, 406, 122 Arp, H. 1965, Science, 148, 363

Barone, L. T., Heithausen, A. H., Fritz, T., \& Klein, U. 2000, MNRAS, 317, 649 Beckwith, S., Sargent, A. I., Chini, R., \& Güsten, R. 1990, AJ, 99, 924

Bendo, G. J., Wilson C. D., Pohlen, M., et al. 2010, A\&A, 518, L65

Cortese, L., Bendo, G. J., Isaak, K. G., Davies, J. I., \& Kent, B. R. 2010, MNRAS, 403, L26

Dirsch, B., Richtler, T., \& Bassino, L. 2003, A\&A, 408, 929

Dirsch, B., Richtler, T., \& Gomez, M. 2005, AJ, 130, 1141

Davies, J. I., Wilson, C. D., Auld, R., et al. 2010, MNRAS, 409, 102

de Mello, D. F., Smith, L. J., Sabbi, E., et al. 2008, AJ, 135, 548

de Vries, H. W., Heithausen, A., \& Thaddeus, P. 1987, ApJ, 319, 723

Engelbracht, C. W., Gordon, K. D., Bendo, G. J., et al. 2004, ApJS, 154, 248

Gordon, K. D., Engelbracht, C. W., Fadda, D., et al. 2007, PASP, 119, 1019

Griffin, M. J., Abergel, A., Abreu, A., et al. 2010, A\&A, 518, L3

Heithausen, A., Stacy, J. G., de Vries, H. W., Mebold, U., \& Thaddeus, P. 1993, A\&A, 268, 265

Heithausen, A. 2002, A\&A, 393, L41

Heithausen, A. 2004, ApJ, 606, L13

Heithausen, A. 2006, A\&A, 450, 193

Heithausen, A., \& Walter, F. 2000, A\&A, 361, 500

Kennicutt, R. C., Armus, L. Bendo, G., et al. 2003, PASP, 115, 928 (SINGS)

Magnani, L., Hartmann, D., Holcomb, S. L., Smith, L. E., \& Thaddeus, P. 2000, ApJ, 535, 167

Miville-Deschênes, M. A., Martin, P. G., Abergel, A., et al. 2010, A\&A, 518, L104

Onishi, T., Yoshikawa, N., Yamamoto, H., et al. 2001, PASJ, 53, 1017

Rieke, G. H., E. T., Young, E. T., Engelbracht C. W., et al. 2004, ApJS, 154, 25 Sandage, A. 1976, AJ, 81, 954

Sollima, A., Gil de Paz, A., Martinez-Delgado, D., et al. 2010, A\&A, 516, A83

Sun, W. H., Zhou, X., Chen, W. P., et al. 2005, ApJ, 630, L133

Sipthorpe, B., Ferlet, M., Bendo, G., et al. 2011, ftp://ftp.sciops.esa. int/pub/hsc-calibration/SPIRE/PHOT/Beams/beam_release_ note_v1-1.pdf

Sodroski, T. J., Odegard, N., Arendt, R. G., et al. 1997, ApJ, 480, 173

SPIRE Observor's Manual 2011,

http://herschel.esac.esa.int/Docs/SPIRE/html/spire_om.html Stansberry, J. A., Gordon, K. D., Bhattacharya, B., et al. 2007, PASP, 119, 1038 Walter, F., \& Heithausen, A. 1999, ApJ, 519, L69

Walter, F., Brinks, E., de Blok, W. J. G., et al. 2008, AJ, 136, 2563

Walter, F., Sandstrom, K., Aniano, G., et al. 2011, ApJ, 726, L11

Ward-Thompson, D., Kirk, J. M., André, P., et al. 2010, A\&A, 518, L92

Wilson, C. D. 1995, ApJ, 448, L97

Xilouris, E., Alton, P., Alikakos, J., et al. 2006, ApJ, 651, L107

Yun, M. S., Ho, P. T. P., \& Lo, K. Y. 1994, Nature, 372, 530 